\title{
Correlation of morphological fraction with redshift in galaxy clusters
}

\author{
Qi-Rong Yuan ${ }^{1}$, Qiang-Qiang Dang ${ }^{1}$, Peng-Fei Yan $^{1}$, Wei Chen ${ }^{1}$, \\ Zhong-Lue Wen ${ }^{2}$, Jin-Lin $\mathrm{Han}^{2}$ and $\mathrm{Xu} \mathrm{Zhou}{ }^{2}$ \\ ${ }^{1}$ Physics Department, Nanjing Normal University, \\ 1 WenYuan Road, Nanjing 210046, China \\ email: yuanqirong@njnu.edu.cn \\ ${ }^{2}$ National Astronomical Observatories, Chinese Academy of Science, \\ 20A Datun Road, Beijing 100012, China
}

\begin{abstract}
Based on 187 galaxy clusters identified from the photometric redshifts of galaxies in the Cosmic Evolution Survey (COSMOS) field(Wen \& Han 2011), cluster galaxies brighter than $M_{V}=-20.5$ are classified into four categories according to their best-fitting templates of the spectral energy distributions (SEDs) provided by Ilbert et al. (2009): early-type (including elliptical and lenticular) galaxies (E+S0), spiral galaxies (S), irregular galaxies (Irr), and starbursts (SB). The fractions of these four SED types are presented as the functions of redshift in Figure 1. Fraction of each category varies remarkably with cluster redshift: fractions of normal galaxies $(\mathrm{E}+\mathrm{S} 0+\mathrm{S}+\mathrm{Irr})$ tend to decrease with redshift, whilst the starburst proportion tends to increase with redshift. For the normal galaxies, there exists a sequence for the decreasing slopes of morphological fractions. Majority of the galaxies in high-redshift clusters $(z>1.0)$ are experiencing strong star-formation activities, which leads to a very high proportion of starburst.
\end{abstract}

Keywords. galaxies: classification, galaxies: evolution, galaxies: clusters: general
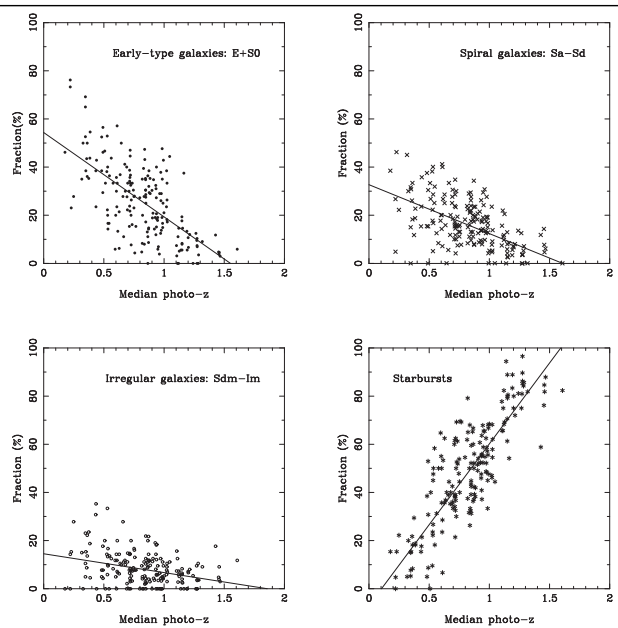

Figure 1. Fractions of four SED categories as the functions of redshift.

This work was supported by National Natural Science Foundation of China (No. 11173016).

\section{References}

Ilbert, O., et al. 2009, ApJ, 690, 1236

Wen, Z. \& Han, J. 2011, ApJ, 724, 68 